\title{
Generalized Quantum Spins, Coherent States, and Lieb Inequalities *
}

\author{
W. Fuller ${ }^{1}$ and A. Lenard ${ }^{2}$ \\ 1 Holy Cross Junior College, Notre Dame, Indiana 46616, USA \\ 2 Indiana University, Bloomington, Indiana 47401, USA
}

\begin{abstract}
A mathematical generalization of the concept of quantum spin is constructed in which the role of the symmetry group $\mathrm{O}_{3}$ is replaced by $\mathrm{O}_{v}$ $(v=2,3,4, \ldots)$. The notion of spin direction is replaced by a point on the manifold of oriented planes in $\mathbb{R}^{v}$. The theory of coherent states is developed, and it is shown that the natural generalizations of Lieb's formulae connecting quantum spins and classical configuration space hold true. This leads to the Lieb inequalities [1] and with it to the limit theorems as the quantum spin $l$ approaches infinity. The critical step in the proofs is the validity of the appropriate generalization of the Wigner-Eckart theorem.
\end{abstract}

\section{Introduction}

The study of the classical limit of certain quantum mechanical systems was the subject of an interesting paper of Lieb [1]. Among the systems considered there is the Heisenberg model whose Hamiltonian is of the form

$$
H_{\text {quant }}=\sum g_{j k} \mathbf{L}^{(j)} \cdot \mathbf{L}^{(k)},
$$

where the $\mathbf{L}^{(j)}$ are independent quantum spins with the same total spin quantum number $l$. To this model corresponds a classical analogue, defined formally by the same energy function with the $\mathbf{L}^{(j)}$ interpreted as vectors of length $l$ in 3dimensional space. If one writes $\mathbf{L}^{(j)}=l \boldsymbol{\omega}^{(j)}$, the $\boldsymbol{\omega}^{(j)}$ unit vectors, the classical model is then defined by its energy function

$$
H_{\text {class }}=l^{2} \sum g_{j k} \omega^{(j)} \cdot \omega^{(k)} .
$$

From the point of view of statistical mechanics one is interested in the respective partition functions

$$
Z_{\text {quant }}(l)=\frac{1}{(2 l+1)^{n}} \operatorname{Tr} \exp \left(-H_{\text {quant }}\right)
$$

* This paper is based largely on the Indiana University Ph. D. thesis of the first named author 
and

$$
Z_{\text {class }}(l)=\frac{1}{(4 \pi)^{n}} \int \ldots \int \exp \left(-H_{\text {class }}\right) d \omega_{1} \ldots d \omega_{n},
$$

where $n$ is the total number of spins and the integral in the classical case is with respect to uniform surface measure (solid angle) over the $n$ unit spheres. Lieb proves two striking inequalities

$$
Z_{\text {class }}(l) \leqq Z_{\text {quant }}(l),
$$

and

$$
Z_{\text {quant }}(l) \leqq Z_{\text {class }}(l+1) \text {. }
$$

They show that as $l$ increases, and the coupling constants $g_{j k}$ are fixed, the quantum and classical partition functions form two non-decreasing and interlacing sequences. An ingenious application of this fact is the theorem, proved by Lieb, that if the coupling constants are scaled inversely proportionally to $l^{2}$ and the limit $l \rightarrow \infty$ is taken after a bulk (thermodynamic) limit, then the free energy per spin in the quantum model goes over into that of the classical model. This is one mathematically precise version of the traditional wisdom that quantum physics goes over into classical physics "in the limit of large quantum numbers".

In the present paper we wish to contribute to a better understanding of how such remarkable results come about. Ingredients of the mathematics leading to (1.5) and (1.6) are familiar to practitioners of statistical mechanics, namely the Peierls-Bogoliubov and Golden-Thompson inequalities. But the possibility of applying these inequalities to the problem at hand depends on certain formulae which relate quantum spin operators to the "classical" unit vectors. These formulae are arrived at by consideration of a distinguished family of quantum states ("coherent states"), definable as eigenvectors of spin components in various directions belonging to their largest positive eigenvalue $l$ (this definition is obscured in the Lieb paper by the use of the inherently unsymmetrical spherical polar coordinate system). Let $\boldsymbol{\omega}=\left(\omega_{1}, \omega_{2}, \omega_{3}\right)$ be a unit vector (direction) in $\mathbb{R}^{3}$, $\mathbf{L}=\left(L_{1}, L_{2}, L_{3}\right)$ a spin vector with the standard commutation rules $L_{1} L_{2}-L_{2} L_{1}$ $=i L_{3}$, etc., $\mathbf{L} \cdot \mathbf{L}=L_{1}^{2}+L_{2}^{2}+L_{3}^{2}=l(l+1), L_{\omega}=\boldsymbol{\omega} \cdot \mathbf{L}=\omega_{1} L_{1}+\omega_{2} L_{2}+\omega_{3} L_{3}$. Then the coherent state $\phi_{\omega}$ belonging to the direction $\omega$ is defined by

$$
L_{\omega} \phi_{\omega}=l \phi_{\omega}
$$

The first of the formulae referred to above expresses the expectation value of $L_{\omega^{\prime}}$ in the state $\phi_{\omega}$, where $\omega$ and $\omega^{\prime}$ are any two directions:

$$
\frac{\left(\phi_{\omega}, L_{\omega^{\prime}} \phi_{\omega}\right)}{\left(\phi_{\omega}, \phi_{\omega}\right)}=l \omega \cdot \omega^{\prime} .
$$

This is the formula at the root of the inequality (1.5). The other inequality (1.6) is based on a more spohisticated fact, namely that the operator $L_{\omega^{\prime}}$ itself may be given an integral representation over the "classical configuration space", the unit sphere of directions $\omega$. This integral representation is

$$
L_{\omega^{\prime}}=(l+1) \frac{2 l+1}{4 \pi} \int \omega^{\prime} \cdot \omega E_{\omega} d \omega,
$$


where $E_{\omega}$ is the projection operator into the one-dimensional space generated by $\phi_{\omega}$, and where the integral is the usual uniform one with respect to the unit vector $\omega$. The occurence of $l+1$ in (1.6) has its origin in the appearance of this factor in the formula (1.9).

There is another integral representation, simpler than (1.9), namely,

$$
I=\frac{2 l+1}{4 \pi} \int E_{\omega} d \omega,
$$

where $I$ is the identity operator. This one implies, in particular, that for any operator $A$

$$
\begin{aligned}
\operatorname{Tr} A & =\frac{2 l+1}{4 \pi} \int \operatorname{Tr}\left(A E_{\omega}\right) d \omega \\
& =\frac{2 l+1}{4 \pi} \int \frac{\left(\phi_{\omega}, A \phi_{\omega}\right)}{\left(\phi_{\omega}, \phi_{\omega}\right)} d \omega .
\end{aligned}
$$

The proofs given by Lieb are basically verifications, starting with the expression for $\phi_{\omega}$ in terms of the spherical polar coordinates of $\boldsymbol{\omega}$. While this is logically without blemish, we feel that it does not sufficient justice to the matter, especially the role of symmetry under orthogonal transformations. We have therefore set ourselves the task of generalizing the whole scheme, in such a manner that on the one hand the quantum-classical analogy should prevail, and that on the other hand the true essence of the formulae analogous to (1.7)-(1.11) shall be revealed. In pursuing a generalization, the obvious first idea suggests itself to replace the "classical" unit sphere in $\mathbb{R}^{3}$ by the unit sphere in a higher dimensional space $\mathbb{R}^{v}(v>3)$. This idea founders on the obstacle that then no reasonable replacement for the operator family $L_{\omega}$ appears possible. Fortunately, there is a way out of this difficulty. It is only necessary to reinterpret the geometrical essence of the situation in $\mathbb{R}^{3}$ slightly, to make the generalization to $\mathbb{R}^{v}$ possible, indeed almost obvious. A direction in $\mathbb{R}^{3}$ is a unit vector, but equally well a plane, the plane orthogonal to the vector. To make this unambiguous, the plane must be assigned an internal sense of positive rotation (orientation), and a convention must be made how the orientation picks out one of the two possible orthogonal unit vectors. Thus the unit sphere $S^{2}$ in $\mathbb{R}^{3}$ may be thought of as the manifold of oriented planes through the origin. This concept makes sense in $\mathbb{R}^{4}, \mathbb{R}^{5}$, etc., indeed already in $\mathbb{R}^{2}$, so that we take the point of view that "classical configuration space" is the manifold of oriented planes (two-dimensional linear subspaces) of $\mathbb{R}^{v}$. With this as a starting point, the theory of quantum spins, coherent states, and so forth, can be developed exactly along the lines of the case $v=3$ familiar from ordinary quantum mechanics. As we shall see, the properly conceived analogues of Lieb's formulae hold for the generalization. The mathematics we develop suggests new statistical mechanical models that may perhaps be of interest on their own.

\section{The Space of Oriented Planes}

A plane $\sigma$ passing through the origin in $\mathbb{R}^{v}(v \geqq 2)$ is determined by two orthogonal unit vectors $\mathbf{a}, \mathbf{b}$ lying in it. An orientation of $\sigma$ is a distinguished sense of rotation in it. This can be specified as the rotation that carries $\mathbf{a}$ into $\mathbf{b}$ by the angle $\pi / 2$. 
Thus $\sigma$ as an oriented plane is completely determined by the ordered pair (a,b). Two ordered pairs $(\mathbf{a}, \mathbf{b})$ and $\left(\mathbf{a}^{\prime}, \mathbf{b}^{\prime}\right)$ determine the same oriented plane $\sigma$ if

$$
\left\{\begin{array}{l}
\mathbf{a}^{\prime}=\mathbf{a} \cos \theta+\mathbf{b} \sin \theta \\
\mathbf{b}^{\prime}=-\mathbf{a} \sin \theta+\mathbf{b} \cos \theta
\end{array}\right.
$$

for a suitable angle $\theta$. Thus an oriented plane is defined precisely as the equivalence class, with respect to the relation (2.1), of ordered pairs $(\mathbf{a}, \mathbf{b})$ of two orthogonal unit vectors.

The set of all oriented planes $\sigma$ in $\mathbb{R}^{v}$ will be denoted $M_{v}$, or simply $M . M_{2}$ has two elements, $\mathbb{R}^{2}$ with its two orientations. $M_{v}$ for $v \geqq 3$ forms a real analytic manifold of dimension $(v-1)+(v-2)-1=2 v-4$. The simplest of these, $M_{3}$, can be identified with the unit sphere $S^{2}$ in $\mathbb{R}^{3}$, for an oriented plane $\sigma$ in $\mathbb{R}^{3}$ determines, and is determined by, a unique unit vector $\mathbf{a} \times \mathbf{b}$ orthogonal to it.

A function $f=f(\mathbf{a}, \mathbf{b})$ may be regarded as defined on $M$ whenever (2.1) implies $f(\mathbf{a}, \mathbf{b})=f\left(\mathbf{a}^{\prime}, \mathbf{b}^{\prime}\right)$, in which case we write $f=f(\sigma)$.

The skew-symmetric matrix $m$ defined by

$$
m_{j k}=a_{j} b_{k}-b_{j} a_{k} \quad(j, k=1,2, \ldots, v)
$$

is invariant with respect to transformations of the form (2.1), hence we may write $m=m(\sigma)$. It has rank 2 , and is so normalized that

$$
\frac{1}{2} \operatorname{Tr}\left(m^{t} m\right)=\sum_{j<k} m_{j k}^{2}=1 .
$$

Conversely, if $m$ is skew-symmetric, has rank2, and satisfies (2.3), then there is a pair $(\mathbf{a}, \mathbf{b})$ of orthogonal unit vectors, determined up to transformations (2.1), such that in terms of it $m$ is given by (2.2). Thus $M$ may be viewed alternately as the space of normalized skew-symmetric matrices of rank 2. From this point of view, $M_{2}$ is thought of as the set of the two matrices $m_{ \pm}=\left(\begin{array}{rr}0 & \pm 1 \\ \mp 1 & 0\end{array}\right)$, while $M_{3}$ is the set of matrices of the form

$$
\left(\begin{array}{rrr}
0 & \omega_{3} & -\omega_{2} \\
-\omega_{3} & 0 & \omega_{1} \\
\omega_{2} & -\omega_{1} & 0
\end{array}\right)
$$

where $\boldsymbol{\omega}=\mathbf{a} \times \mathbf{b}$ varies over unit vectors in $\mathbb{R}^{3}$.

The orthogonal group $O=O_{v}$ acts in a natural manner on $M_{v}$. Let $R \in O_{v}$ be an orthogonal matrix, and write $R \mathbf{x}$ for the vector that it transforms any $\mathbf{x} \in \mathbb{R}^{v}$ into. If (a,b) determines the oriented plane $\sigma$, we write $R \sigma$ for the oriented plane determined by $(R \mathbf{a}, R \mathbf{b})$. The transformation law for the corresponding matrices is

$$
m(R \sigma)=R m(\sigma) R^{t},
$$

where the superscript $t$ denotes matrix transpose. The action of $O_{v}$ on $M_{v}$ is transitive: any $\sigma \in M_{v}$ may be transformed into any $\sigma^{\prime} \in M_{v}$ by a suitable $R \in O_{v}$. Thus $M_{v}$ under the action of $O_{v}$ is a homogeneous space and, as such, may be viewed yet another way, namely as arising from the group manifold of $O_{v}$ by a suitable identification process; but we do not emphasize this aspect. The action of 
$\mathrm{O}_{2}$ on $M_{2}$ is quite trivial, in that $\mathrm{R} \in \mathrm{O}_{2}$ either leaves the two elements of $M_{2}$ invariant (Det $R=1$ ), or interchanges them (Det $R=-1$ ). The action of $O_{3}$ on $M_{3}$ may be expressed in terms of the normal vector $\omega$ uniquely associated with $\sigma$ as follows : $\omega(R \sigma)=(\operatorname{Det} R) R \omega(\sigma)$.

Let $\sigma, \sigma^{\prime}$ be any two oriented planes of $M_{v}$. Let $F$ be any geometric figure in $\sigma$ of positive 2-dimensional Lebesgue measure $A(F)$. If $F^{\prime}$ is the orthogonal projection of $F$ onto $\sigma^{\prime}$, we denote by $\varrho_{v}$ the ratio $\pm A\left(F^{\prime}\right) / A(F)$, taken positively if projection preserves orientation, negatively if it reverses it. $\varrho_{v}$ clearly depends only on $\sigma$ and $\sigma^{\prime}$ and it has the properties $-1 \leqq \varrho_{v}\left(\sigma, \sigma^{\prime}\right) \leqq 1, \varrho_{v}\left(\sigma, \sigma^{\prime}\right)=\varrho_{v}\left(\sigma^{\prime}, \sigma\right), \varrho_{v}\left(R \sigma, R \sigma^{\prime}\right)=\varrho_{v}\left(\sigma, \sigma^{\prime}\right)$ for all $\sigma, \sigma^{\prime} \in M_{\nu}$ all $R \in O_{v}$. The explicit formula for $\varrho_{v}$ is

$$
\begin{aligned}
\varrho_{v}\left(\sigma, \sigma^{\prime}\right) & =\left(\mathbf{a} \cdot \mathbf{a}^{\prime}\right)\left(\mathbf{b} \cdot \mathbf{b}^{\prime}\right)-\left(\mathbf{a} \cdot \mathbf{b}^{\prime}\right)\left(\mathbf{b} \cdot \mathbf{a}^{\prime}\right) \\
& =\frac{1}{2} \operatorname{Tr} m^{t}(\sigma) m\left(\sigma^{\prime}\right) \\
& =\sum_{j<k} m_{j k}(\sigma) m_{j k}\left(\sigma^{\prime}\right)
\end{aligned}
$$

In particular, $\varrho_{2}\left(\sigma, \sigma^{\prime}\right)= \pm 1$ depending on whether $\sigma=\sigma^{\prime}$ or $\neq \sigma^{\prime}$, while $\varrho_{3}\left(\sigma, \sigma^{\prime}\right)$ $=\omega(\sigma) \cdot \boldsymbol{\omega}\left(\sigma^{\prime}\right)$.

Integration over $M_{v}$ is defined in terms of normalized Haar-measure $h=h_{v}$ over $O_{v}$. If $f=f(\sigma)$ is a continuous function on $M_{v}$, its integral is defined by

$$
\int_{M_{v}} f(\sigma) d \mu(\sigma)=\int_{O_{v}} f\left(R \sigma_{0}\right) d h(R),
$$

where $\sigma_{0}$ is some, arbitrarily chosen, plane in $M_{v}$. Since $h\left(O_{v}\right)=1$, it follows that $\mu\left(M_{v}\right)=1$. For $v=2$ "integration" is simply averaging

$$
\int_{M_{2}} f(\sigma) d \mu(\sigma)=\frac{1}{2} f\left(\sigma_{+}\right)+\frac{1}{2} f\left(\sigma_{-}\right),
$$

where $M_{2}=\left\{\sigma_{+}, \sigma_{-}\right\}$. For $v=3$ it is given by the familiar formula

$$
\int_{M_{3}} f(\sigma) d \mu(\sigma)=\frac{1}{4 \pi} \int_{S_{2}} f(\omega) d \omega
$$

in terms of the solid angle element $d \omega$ with respect to the unit normal vector.

\section{Spherical Harmonics and Spin Operators}

We summarize briefly the appropriate generalization of the theory of spin operators, based on a construction in terms of spherical harmonics. For an extensive treatment of spherical harmonics in $\mathbb{R}^{v}$ we refer the reader to Chap. 6 in the book of Hochstadt [2], and to Chap. IX in the book of Vilenkin [3].

A spherical harmonic of degree $l(l=0,1,2, \ldots)$ in $v$ variables $(v=2,3, \ldots)$ is a homogeneous polynomial $f\left(x_{1}, x_{2}, \ldots, x_{v}\right)=f(\mathbf{x})$ of degree $l$ that satisfies Laplace's equation $\Delta f=0$.

The dimension of the linear space of all homogeneous polynomials of degree $l$ is the binomial coefficient $\left(\begin{array}{c}v+l-1 \\ l\end{array}\right)$. Spherical harmonics form a subspace (a 
proper one for $l \geqq 2$ ) of dimension

$$
d=d_{v, l}=\frac{(v+l-3) !(v+2 l-2)}{l !(v-2) !},
$$

a number that reduces to the familiar $2 l+1$ for the case $v=3$. We denote by $\mathfrak{H}=\mathfrak{S}_{v, l}$ the space of spherical harmonics of degree $l$ in $v$ variables. It is made into a Hilbert space by the definition of an inner product

$$
(f, g)=\frac{1}{\Omega} \int \overline{f(\mathbf{x})} g(\mathbf{x}) d \omega,
$$

where integration is over the unit sphere $S^{\nu-1}$, and the element of surface $d \omega$ is so normalized that $r^{v-1} d r d \omega$ is the Euclidean volume element in $\mathbb{R}^{v}$.

$$
\Omega=\Omega_{v}=\frac{2 \Gamma\left(\frac{1}{2}\right)^{v}}{\Gamma(v / 2)}
$$

is the surface measure of the unit sphere. The calculation of inner products in $\mathfrak{H}$ is facilitated by knowing integrals of the form

$$
I\left(k_{1}, \ldots, k_{v}\right)=\frac{1}{\Omega} \int x_{1}^{k_{1}} \ldots x_{v}^{k_{v}} d \omega
$$

where the $k_{j}$ are non-negative integers. $I$ vanishes unless all $k_{j}$ are even, and in that case

$$
I\left(k_{1}, \ldots, k_{j}\right)=\frac{\Gamma\left(\frac{v}{2}\right) \Gamma\left(\frac{k_{1}+1}{2}\right) \ldots \Gamma\left(\frac{k_{v}+1}{2}\right)}{\Gamma\left(\frac{1}{2}\right)^{v} \Gamma\left(\frac{k_{1}+\ldots+k_{v}+v}{2}\right)} .
$$

This formula is obtained by noting that

$$
I\left(k_{1}, \ldots, k_{v}\right) \int_{0}^{\infty} e^{-r^{2}} r^{k_{1}+\ldots+k_{v}+v-1} d r
$$

and

$$
\int_{-\infty}^{\infty} e^{-x_{1}^{2}} x_{1}^{k_{1}} d x_{1} \ldots \int_{-\infty}^{\infty} e^{-x_{v}^{2}} x_{v}^{k_{v}} d x_{v}
$$

are proportional. A related and useful formula is

$$
\frac{1}{\Omega} \int\left(\mathbf{x} \cdot \mathbf{y}_{1}\right)\left(\mathbf{x} \cdot \mathbf{y}_{2}\right) \ldots\left(\mathbf{x} \cdot \mathbf{y}_{2 n}\right) d \omega=\frac{\Gamma(v / 2)}{(n+v / 2) \cdot 2^{n}} P\left(\mathbf{y}_{1}, \ldots, \mathbf{y}_{2 n}\right),
$$

where $P$ is the "pairing sum", the sum of the $(2 n-1) ! !=(2 n-1)(2 n-3) \ldots 3 \cdot 1$ products, formed with $n$ dot-products $\mathbf{y}_{j} \cdot \mathbf{y}_{k}$, in each of which all of vectors $\mathbf{y}_{j}$ occur. This fact is obtained by noting that both sides are multilinear in the $\mathbf{y}_{j}$, symmetric under permutations, and invariant under $\mathbf{y}_{j} \rightarrow R \mathbf{y}_{j}$ with $R \in O_{v}$ arbitrary. This shows that they must be proportional, and the constant of proportionality is checked in the special case $\mathbf{y}_{1}=\ldots=\mathbf{y}_{2 n}=\mathbf{e}_{1}=(1,0,0, \ldots, 0)$. 
In the space $\mathfrak{H}$ a unitary representation $R \rightarrow U(R)$ of $O_{\nu}$ is defined by

$$
U(R) f(\mathbf{x})=f\left(R^{t} \mathbf{x}\right) .
$$

Spin operators acting in $\mathfrak{H}$ are defined in terms of $U$ by the following construction. Let $(\mathbf{a}, \mathbf{b})$ determine the oriented plane $\sigma$. Let $T_{\sigma}^{\theta}$ denote the rotation of $\mathbb{R}^{v}$ specified by

$$
\left\{\begin{array}{l}
T_{\sigma}^{\theta} \mathbf{a}=\mathbf{a} \cos \theta+\mathbf{b} \sin \theta \\
T_{\sigma}^{\theta} \mathbf{b}=-\mathbf{a} \sin \theta+\mathbf{b} \cos \theta \\
T_{\sigma}^{\theta} \mathbf{x}=\mathbf{x} \text { if } \mathbf{x} \cdot \mathbf{a}=\mathbf{x} \cdot \mathbf{b}=0 .
\end{array}\right.
$$

Then we define

$$
L_{\sigma}=\lim _{\theta \rightarrow 0} \frac{1}{i \theta}\left\{U\left(T_{\sigma}^{\theta}\right)-I\right\},
$$

and call it the spin operator associated with $\sigma \in M$. Explicitly

$$
i L_{\sigma}=(\mathbf{a} \cdot \mathbf{x})(\mathbf{b} \cdot \operatorname{grad})-(\mathbf{b} \cdot \mathbf{x})(\mathbf{a} \cdot \operatorname{grad}),
$$

or also

$$
L_{\sigma}=\sum_{j<k} m_{j k}(\sigma) L_{j k}
$$

where

$$
i L_{j k}=x_{j} \frac{\partial}{\partial x_{k}}-x_{k} \frac{\partial}{\partial x_{j}} .
$$

These definitions are straightforward transcriptions of the familiar ones from quantum mechanics (the case $v=3$ ). The only novelty is that "spin direction" is interpreted in the general case as an oriented plane in $\mathbb{R}^{v}$.

Spin operators $L_{\sigma}$ for varying $\sigma \in M$ are related to each other by the natural action of the orthogonal group $O_{v}$. The identity

$$
T_{R \sigma}^{\theta}=R T_{\sigma}^{\theta} R^{-1}
$$

implies

$$
L_{R \sigma}=U(R) L_{\sigma} U^{*}(R),
$$

or equivalently,

$$
L_{\sigma}=U(R) L_{R^{t} \sigma} U^{*}(R),
$$

This identity, the transformation law of spin operators under the action of $O_{v}$, will be of particular importance below. We rewrite it in yet another form in terms of the coordinate-plane spins $L_{j k}$. For any $R \in O_{v}$ define a matrix $W(R)$ with $\left(\begin{array}{l}v \\ 2\end{array}\right)$ rows and columns as follows

$$
W_{j k, r s}(R)=R_{j r} R_{k s}-R_{j s} R_{k r} \quad(1 \leqq j<k \leqq v, 1 \leqq r<s \leqq v) .
$$


Then (3.17) may be rewritten

$$
L_{j k}=\sum_{r<s} W_{j k, r s}(R) U(R) L_{r s} U^{*}(R) .
$$

The correspondence $R \rightarrow W(R)$ is a unitary matrix representation of $O_{v}$ (the antisymmetrized tensor product of the identity representation with itself). One obtains (3.17) from (3.19) upon multiplying with $m_{j k}(\sigma)$, summing over subscripts and making use of (2.4).

Proposition 1. The spherical harmonic

$$
\phi_{a b}(\mathbf{x})=(\mathbf{a} \cdot \mathbf{x}+i \mathbf{b} \cdot \mathbf{x})^{l}
$$

is the unique eigenfunction of $L_{\sigma}$ belonging to its largest positive eigenvalue $l$, satisfying $\phi_{a b}(\mathbf{a})=1$, where $(\mathbf{a}, \mathbf{b})$ determine $\sigma \in M$.

Proof. In view of the transformation law (3.16) it suffices to consider a particular $\sigma$, for instance the one determined by $\left(\mathbf{e}_{1}, \mathbf{e}_{2}\right)$. Every $f=f(\mathbf{x})$ is $\mathfrak{H}$ has the form

$$
f=\sum_{r s} c_{r s}\left(x_{1}+i x_{2}\right)^{r}\left(x_{1}-i x_{2}\right)^{s}
$$

where $c_{r s}=c_{r s}\left(x_{3}, \ldots, x_{v}\right)$ is a homogeneous polynomial of degree $l-r-s$. Since

$$
L_{12} f=\sum_{r s}(r-s) c_{r s}\left(x_{1}+i x_{2}\right)^{r}\left(x_{1}-i x_{2}\right)^{s}
$$

$f$ can be an eigenfunction of $L_{12}$ only if for all non-vanishing $c_{r s}$ the same value is taken by $r-s$; and the largest such value is evidently $l$, when $c_{l 0}$ is a constant. The requirement $f\left(\mathbf{e}_{1}\right)=1$ fixes it uniquely, so that $f(\mathbf{x})=\left(x_{1}+i x_{2}\right)^{l}$, Q.E.D.

The eigenfunction $\phi_{a b}$ of $L_{\sigma}$ is said to determine the coherent state belonging to $\sigma$. Note that as the pair $(\mathbf{a}, \mathbf{b})$ is replaced by the equivalent pair $\left(\mathbf{a}^{\prime}, \mathbf{b}^{\prime}\right)$ via $(2.1), \phi_{a b}$ is replaced by $\phi_{a^{\prime} b^{\prime}}=e^{-i l \theta} \phi_{a b}$. Thus the quantum mechanical state is determined by $\sigma$ alone.

Inner products of coherent state functions are easily computed with the integration formula (3.8). One finds

$$
\left(\phi_{a b}, \phi_{a^{\prime} b^{\prime}}\right)=N^{2}\left(\frac{\mathbf{a} \cdot \mathbf{a}^{\prime}+\mathbf{b} \cdot \mathbf{b}^{\prime}}{2}+i \frac{\mathbf{a} \cdot \mathbf{b}^{\prime}-\mathbf{b} \cdot \mathbf{a}^{\prime}}{2}\right)^{l}=N^{2} \phi_{a^{\prime} b^{\prime}}\left(\frac{\boldsymbol{a}-i \boldsymbol{b}}{2}\right),
$$

where

$$
N=N_{v, l}=\left(\frac{l ! \Gamma(v / 2)}{\Gamma(l+v / 2)}\right)^{1 / 2}
$$

is the norm of $\phi_{a b}$ in the Hilbert space $\mathfrak{H}$.

\section{Integral Representation of Spin Operators in Terms of Coherent States}

We come to the appropriate generalizations of $(1.8)-(1.11)$. The notational convention will be adopted that the pairs $(\mathbf{a}, \mathbf{b})$ and $\left(\mathbf{a}^{\prime}, \mathbf{b}^{\prime}\right)$ determine the oriented planes $\sigma$ and $\sigma^{\prime}$ respectively, and $E_{\sigma}$ is the projection operator onto the ray 
determined by $\phi_{a b}$. Subscripts $v$ and $l$ will be omitted where no confusion is possible.

The analogue of (1.8) is

Proposition 2. For all $\sigma$ and $\sigma^{\prime}$ in $M$,

$$
N^{-2}\left(\phi_{a b}, L_{\sigma^{\prime}} \phi_{a b}\right)=l \varrho\left(\sigma, \sigma^{\prime}\right) \text {. }
$$

Proof. Use (3.12) and (3.20) for $L_{\sigma^{\prime}}$ and $\phi_{a b}$ respectively, calculate the inner product (3.2) via (3.8), and compare the result with (2.6), Q.E.D.

The integral representation of the identity operator, generalization of (1.10), is

\section{Proposition 3.}

$$
I=d \int_{M} E_{\sigma} d \mu(\sigma) .
$$

Proof. It is only necessary to show that the integral is a multiple of $I$, since the proportionality constant is established by taking the trace and noting $\operatorname{Tr} E_{\sigma}=1$. This is accomplished as soon as it is shown that the integral commutes with each $U(R), R \in O_{v}$, because $U$ is irreducible. (See Vilenkin [3] for proof. In cases $v=2$, $l \geqq 1, U$ is reducible when viewed as a representation of the subgroup $\mathrm{SO}_{2}$ of rotations, but still irreducible when, as here, viewed as a representation of the full orthogonal group $\mathrm{O}_{2}$ that includes reflections.) For every $R \in O_{v}$ and every $(\mathbf{a}, \mathbf{b})$

$$
U(R) \phi_{a b}=\phi_{R a R b}
$$

so that

$$
\left(\phi_{R a R b}, U(R) f\right)=\left(\phi_{a b}, f\right)
$$

for all $f \in \mathfrak{H}$. A straightforward calculation then shows that $U(R) E_{\sigma} f=E_{R \sigma} U(R) f$, in other words

$$
E_{R \sigma} U(R)=U(R) E_{\sigma} .
$$

If this is integrated over $M$ with respect to the measure $\mu$, being invariant with respect to the action $\sigma \rightarrow R_{\sigma}$ of $O_{v}$, one obtains the desired equation

$$
\int_{M} E_{\sigma} d \mu(\sigma) U(R)=U(R) \int_{M} E_{\sigma} d \mu(\sigma) \text {, Q.E.D. }
$$

Of course, there is an analogue of the trace formula (1.11).

Proposition 4. For any operator $A$ acting in $\mathfrak{H}, \operatorname{Tr} A=d N^{-2} \int_{M}\left(\phi_{a b}, A \phi_{a b}\right) d \mu(\sigma)$.

Proof. Write $A=A I$, use the integral representation for $I$, note $\operatorname{Tr}\left(A E_{\sigma}\right)=N^{-2}$ $\left(\phi_{a b}, A \phi_{a b}\right)$, Q.E.D.

The only difficult one among the facts to be established is the generalization of (1.9), the integral representation of spin operators. It is

Proposition 5. For all $\sigma^{\prime} \in M, L_{\sigma^{\prime}}=(l+v-2) d \int_{M} \varrho\left(\sigma^{\prime}, \sigma\right) E_{\sigma} d \mu(\sigma)$.

The rest of this section will be devoted to its proof. 
To make the proof more comprehensible, we outline the steps to be taken. First we show that $L_{\sigma^{\prime}}$ and the operator represented by the integral behave the same way under conjugation with the unitary operators $U(R), R \in O_{v}$. Then, and this is the critical step, we prove that this behavior uniquely determines the family of operators in question up to a multiplicative numerical factor. This statement is the analogue of the Wigner-Eckart theorem in quantum mechanics. Finally, we compute the proportionality constant and show that it is $(l+v-2) d$.

Let $T_{\sigma^{\prime}}$ denote the integral that occurs in Proposition 5. Using (4.3), one obtains, after a simple calculation

$$
T_{R \sigma^{\prime}}=U(R) T_{\sigma^{\prime}} U^{*}(R) .
$$

This is the same equation as (3.16) if $T$ is changed to $L$. Write $T_{\sigma}=\sum_{j<k} m_{j k}(\sigma) T_{j k}$, so that

$$
T_{j k}=\int_{M} m_{j k}(\sigma) E_{\sigma} d \mu(\sigma) .
$$

Then the $T_{j k}$ satisfy the Eq. (3.19). Let us rewrite the latter in matrix form, obtained by expressing operators as matrices with respect to some fixed orthonormal basis $\left\{f_{1}, f_{2}, \ldots, f_{d}\right\}$ in $\mathfrak{H}$. Let $A_{\alpha \beta}=\left(f_{\alpha}, A f_{\beta}\right)(1 \leqq \alpha, \beta \leqq d)$ for any operator $A$ acting in $\mathfrak{H}$. The equation reads

$$
\xi_{j k \alpha \delta}=\sum_{r<s} \sum_{\beta \gamma} W_{j k, r s}(R) U_{\alpha \beta}(R) \bar{U}_{\delta \gamma}(R) \xi_{r s \beta \gamma} \quad \text { (for all } \quad R \in O_{v} \text { ). }
$$

It is satisfied by both $\xi=L$ and $\xi=T$. We intend to show that the solution space is one dimensional, whence it will follow that the $L_{j k}$ are proportional to the $T_{j k}$.

The product of the first two factors under the sum in (4.7) forms a matrix entry of $W(R) \otimes U(R)$, a matrix of $\left(\begin{array}{l}v \\ 2\end{array}\right) d$ rows and columns. The correspondence $R \rightarrow W(R) \otimes U(R)$ is a unitary representation of $O_{v}$. As such, it is equivalent to a direct sum of irreducible representations

$$
W(R) \otimes U(R)=S\left(\begin{array}{cccc}
V^{1}(R) & 0 & \ldots & 0 \\
0 & V^{2}(R) & \ldots & 0 \\
\ldots \ldots \ldots \ldots \ldots \ldots \ldots \ldots \ldots \\
0 & 0 & \ldots & V^{q}(R)
\end{array}\right) S^{*},
$$

where the $V^{\tau}(R)(1 \leqq \tau \leqq q)$ are irreducible unitary representations of $O_{v}$ and $S$ is some unitary matrix. We adopt the convention that any $V^{\tau}$ equivalent to $U$ is taken actually equal $U$; this can clearly be arranged by choosing $S$ suitably. In terms of matrix entries (4.8) reads

$$
W_{j k, r s}(R) U_{\alpha \beta}(R)=\sum_{\tau} \sum_{\eta \varepsilon} S_{j k \alpha, \tau \eta} V_{\eta \varepsilon}^{\tau}(R) \bar{S}_{r s \beta, \tau \varepsilon} .
$$

Substitute into (4.7), integrate over $O_{v}$ with respect to normalized Haar measure, and make use of the orthogonality relations of the matrix entries of unitary 
representations $([3]$, p. 45). This yields

$$
\xi_{j k \alpha \delta}=d^{-1} \sum_{\tau}^{\prime} S_{j k \alpha, \tau \delta} \sum_{r<s} \sum_{\beta \gamma} \bar{S}_{r s \beta, \tau \gamma} \xi_{r s \beta \gamma}=\sum_{\tau}^{\prime} c_{\tau} S_{j k \alpha, \tau \delta},
$$

where the sum $\sum^{\prime}$ is restricted to those terms for which $V^{\tau}=U$. We see that the dimension of the solution space of (4.7) does not exceed the number of terms in the right hand side of (4.10), i.e. the number of times the irreducible representation $U$ occurs in the direct sum decomposition of the representation $W \otimes U$ into irreducibles.

Lemma. For $v \geqq 2$ and $l \geqq 1$ the multiplicity of $U$ in the direct sum decomposition of $W \otimes U$ is 1 .

This will be proved in Sect. 5. In the mean time we can conclude that (4.7) has a unique solution up to a numerical factor. Therefore there is a number $c=c(v, l)$ such that $L_{\sigma}=c T_{\sigma}$ for all $\sigma \in M$. It may be remarked that this conclusion remains valid, even if trivially, in the case $l=0$ not covered by the Lemma, for in that case $L_{\sigma}$ as well as $T_{\sigma}$ vanish.

It remains to determine $c$. Consider the integral

$$
\int_{M} \varrho\left(R \sigma, \sigma^{\prime}\right) \operatorname{Tr}\left(E_{R_{\sigma}} E_{\sigma^{\prime}}\right) d \mu\left(\sigma^{\prime}\right)
$$

as $R$ varies in $O_{v}$. It is easily seen to be independent of $R$, so that we can write

$$
\int_{M} \varrho\left(\sigma, \sigma^{\prime}\right) \operatorname{Tr}\left(E_{\sigma} E_{\sigma^{\prime}}\right) d \mu\left(\sigma^{\prime}\right)=k,
$$

where $k$ is independent of $\sigma$. Thus

$$
\operatorname{Tr} \sum_{j<k} L_{j k}^{2}=c^{2} \sum_{j<k} \operatorname{Tr}\left(T_{j k}^{2}\right)=c^{2} \int_{M} \int_{M} \varrho\left(\sigma, \sigma^{\prime}\right) \operatorname{Tr}\left(E_{\sigma} E_{\sigma^{\prime}}\right) d \mu(\sigma) d \mu\left(\sigma^{\prime}\right)=c^{2} k
$$

But

$$
\sum_{j<k} L_{j k}^{2}=-|\mathbf{x}|^{2} \Delta+(\mathbf{x} \cdot \operatorname{grad})^{2}+(v-2)(\mathbf{x} \cdot \operatorname{grad})=l(l+v-2) I
$$

so that

$$
c^{2} k=l(l+v-2) d
$$

To eliminate $k$, substitute $\mathbf{x}=\frac{1}{2}(\mathbf{a}-i \mathbf{b})$ into $N^{-2}\left(\phi_{a^{\prime} b^{\prime}}, \phi_{a b}\right) \phi_{a^{\prime} b^{\prime}}(\mathbf{x})=E_{\sigma^{\prime}} \phi_{a b}(\mathbf{x})$, so that in view of (3.23) one obtains

$$
E_{\sigma^{\prime}} \phi_{a b}\left(\frac{\boldsymbol{a}-i \boldsymbol{b}}{2}\right)=N^{-2}\left|\left(\phi_{a^{\prime} b^{\prime}}, \phi_{a b}\right)\right|^{2}=\operatorname{Tr}\left(E_{\sigma} E_{\sigma^{\prime}}\right)
$$

When this is multiplied by $c \varrho\left(\sigma, \sigma^{\prime}\right)$ and integrated with respect to $\sigma^{\prime}$, the result is $c T_{\sigma} \phi_{a b}\left(\frac{\boldsymbol{a}-i \boldsymbol{b}}{2}\right)=c k$. But $c T_{\sigma}=L_{\sigma}, L_{\sigma} \phi_{a b}=l \phi_{a b}$, and $\phi_{a b}\left(\frac{\boldsymbol{a}-i \boldsymbol{b}}{2}\right)=1$. This shows that $c k=l$, so that $c=(l+v-2) d$ as was to be shown. Proposition 5 now only depends on the truth of the lemma. 


\section{Proof of the Lemma}

The multiplicity of the irreducible representation $U$ in the direct sum decomposition in terms of irreducibles of the representation $W \otimes U$ is computed by means of the orthogonality relations of group characters, i.e. the traces of the representing matrices. If $V^{\tau}(R)$ and $V^{\tau^{\prime}}(R)$ are two inequivalent irreducible unitary representations of $O_{v}$ then

$$
\int_{o_{v}} V_{\alpha \beta}^{\tau}(R) V_{\gamma \delta}^{\tau^{\prime}}(R) d h(R)=0
$$

and

$$
\int_{O_{v}} \overline{V_{\alpha \beta}^{\tau}}(R) V_{\gamma \varepsilon}^{\tau}(R) d h(R)=\frac{1}{d_{\tau}} \delta_{\alpha \gamma} \delta_{\beta \varepsilon},
$$

where $d_{\tau}$ is the dimension of the representation space. Therefore

$$
\int_{O_{v}} \overline{\operatorname{Tr} V^{\tau}(R)} \operatorname{Tr} V^{\tau^{\prime}}(R) d h(R)=\left\{\begin{array}{l}
0 \\
1
\end{array}\right.
$$

depending on whether $V^{\tau}$ and $V^{\tau^{\prime}}$ are inequivalent or equivalent respectively. It follows that the required multiplicity is

$$
\int_{o_{v}} \operatorname{Tr} W(R) \otimes U(R) \overline{\operatorname{Tr} U(R)} d h(R)=\int_{O_{v}} \operatorname{Tr} W(R)|\operatorname{Tr} U(R)|^{2} d h(R) .
$$

It must be shown that this integral has the value 1 (for $l \geqq 1$ ).

The trace of $W$ is easily calculated from the definition (3.18)

$$
\begin{aligned}
\operatorname{Tr} W(R) & =\sum_{j<k} W_{j k, j k}(R) \\
& =\frac{1}{2} \sum_{j, k} W_{j k, j k}(R) \\
& =\frac{1}{2}(\operatorname{Tr} R)^{2}-\frac{1}{2} \operatorname{Tr}\left(R^{2}\right) .
\end{aligned}
$$

The computation of the trace of $U$ is more complicated, but there is an elegant procedure for obtaining it which, for the sake of completeness, is outlined below. Since various values of $l$ become relevant, this parameter will be displayed explicitly. Thus we write $\mathfrak{H}_{l}$ for the space of spherical harmonics of degree $l, U_{l}$ for the corresponding representation of $O_{v}$. The larger space $\mathfrak{H}_{l}^{\prime}$ of all homogeneous polynomials of degree $l$ (harmonic or not) is also needed. A natural representation $U^{\prime}$ of $\mathrm{GL}_{v, \mathbb{C}}$ (the group of complex non-singular $v \times v$ matrices) is given by $U_{l}^{\prime}(R) f(\mathbf{x})=f\left(R^{-1} \mathbf{x}\right)$ for $R \in \mathrm{GL}_{v, \mathbb{C}}$ and $f \in \mathfrak{H}_{l}^{\prime}$. Here $\mathfrak{H}_{l}^{\prime}$ is regarded merely as a complex linear space so that $U_{l}^{\prime}(R)$ need not, and in general will not, be unitary.

When $R_{1}$ and $R_{2}$ are conjugate in $\mathrm{GL}_{v, \mathbb{C}}, R_{1}=R R_{2} R^{-1}$ for some $R$, then $\operatorname{Tr} U_{l}^{\prime}\left(R_{1}\right)=\operatorname{Tr} U_{l}^{\prime}\left(R_{2}\right)$. It suffices then to obtain the trace when $R$ is in Jordan canonical form. Since $\operatorname{Tr} U_{l}^{\prime}(R)$ depends continuously on $R$, it is even enough to assume that $R$ has distinct eigenvalues and therefore is a diagonal matrix with non-vanishing diagonal entries $r_{1}, r_{2}, \ldots, r_{v}$. A basis in $\mathfrak{H}_{l}^{\prime}$ is provided by the monomials $f(\mathbf{x})=x_{1}^{k_{1}} x_{2}^{k_{2}} \ldots x_{v}^{k_{v}}$ with the $k_{j} \geqq 0$ integers, $\sum k_{j}=l$. They are eigenfunctions of $U_{l}^{\prime}(R)$ with respective eigenvalues $r_{1}^{-k_{1}} r_{2}^{-k_{2}} \ldots r_{v}^{-k_{v}}$. Therefore

$$
\operatorname{Tr} U_{l}^{\prime}(R)=\sum_{k_{1}+\ldots+k_{v}=l} r_{1}^{-k_{1}} \ldots r_{v}^{-k_{v}}
$$


and hence

$$
\begin{aligned}
\sum_{l=0}^{\infty} z^{l} \operatorname{Tr} U_{l}^{\prime}(R) & =\left(1-\frac{z}{r_{1}}\right)^{-1} \ldots\left(1-\frac{z}{r_{v}}\right)^{-1} \\
& =\operatorname{Det}\left(1-z R^{-1}\right)^{-1}
\end{aligned}
$$

The coefficients in the power series expansion of the right hand side of (5.7) are customarily denoted $p_{l}=p_{l}(R)$, thus $\operatorname{Tr} U_{l}^{\prime}(R)=p_{l}(R)$. Note that if $R \in O_{v}$ the generating function may equally well be written $\operatorname{Det}(1-z R)^{-1}$.

When $l=0,1, \mathfrak{H}_{l}=\mathfrak{H}_{l}^{\prime}$ so that $\operatorname{Tr} U_{l}(R)=p_{l}(R)$. When $l \geqq 2$, the relevant fact is that every $f \in \mathfrak{H}_{l}^{\prime}$ is of the form $f(\mathbf{x})=g(\mathbf{x})+|\mathbf{x}|^{2} h(\mathbf{x})$ where $g \in \mathfrak{H}_{l}$ and $h \in \mathfrak{H}_{l-2}^{\prime}$ (see Vilenkin [3], p. 444). This means that $\mathfrak{H}_{l}^{\prime}$ is the direct sum of $\mathfrak{H}_{l}$ and $|\mathbf{x}|^{2} \mathfrak{Y}_{l-2}^{\prime}$, a decomposition invariant under $U_{l}^{\prime}(R)$ when $R$ is restricted to $O_{v}$. This shows that $U_{l}^{\prime}(R)$ is the direct sum of $U_{l}(R)$ and $U_{l-2}^{\prime}(R)$, whence

$$
\operatorname{Tr} U_{l}^{\prime}(R)=\operatorname{Tr} U_{l}(R)+\operatorname{Tr} U_{l-2}^{\prime}(R) .
$$

Thus

$$
\operatorname{Tr} U_{l}(R)=p_{l}(R)-p_{l-2}(R) \quad(l \geqq 2) .
$$

In generating function form

$$
\begin{aligned}
& \sum_{l=0}^{\infty} z^{l} \operatorname{Tr} U_{l}(R)=\frac{1-z^{2}}{\operatorname{Det}(1-z R)} . \\
& \left(R \in O_{v}\right) .
\end{aligned}
$$

The first few of the $p_{l}$ are $p_{0}=1, p_{1}=\operatorname{Tr} R, p_{2}=\frac{1}{2} \operatorname{Tr}\left(R^{2}\right)+\frac{1}{2}(\operatorname{Tr} R)^{2}$. Following this pattern, one can express $p_{l}$ as a polynomial with rational coefficients in $\operatorname{Tr} R$, $\operatorname{Tr}\left(R^{2}\right), \ldots, \operatorname{Tr}\left(R^{l}\right)$. This comes from the identity

$$
\operatorname{Det}(1-z R)^{-1}=\exp \{-\operatorname{Tr} \log (1-z R)\}=\exp \sum_{k=1}^{\infty} \frac{z^{k}}{k} \operatorname{Tr}\left(R^{k}\right) .
$$

The trace of $W(R)$, computed above in (5.5), can also be expressed as $p_{1}^{2}(R)-p_{2}(R)$.

With these facts at our disposal the proof of the Lemma is reduced to showing that for $l \geqq 1$

$$
\int_{O_{v}}\left(p_{1}^{2}-p_{2}\right)\left(p_{l}-p_{l-2}\right)^{2} d h(R)=1
$$

(for $l=1$ omit $p_{l-2}$ ).

Consider first the simplest case $v=2 . R \in O_{2}$ is one of the two forms

$$
R=\left(\begin{array}{c}
\cos \theta \sin \theta \\
\mp \sin \theta \pm \cos \theta
\end{array}\right)
$$

depending on Det $R= \pm 1$. Integration with respect to Haar measure over $\mathrm{O}_{2}$ is expressed by

$$
\underbrace{\frac{1}{4 \pi} \int_{0}^{2 \pi} \ldots d \theta}_{\operatorname{Det} R=1}+\underbrace{\frac{1}{4 \pi} \int_{0}^{2 \pi} \ldots d \theta}_{\operatorname{Det} R=-1} .
$$

From (5.13) and obtains $\operatorname{Det}(1-z R)=1-2 z+z^{2}$ when $\operatorname{Det} R=1$, and $1-z^{2}$ when $\operatorname{Det} R=-1$. In view of the expansion

$$
\frac{1-z^{2}}{1-2 z \cos \theta+z^{2}}=1+2 \sum_{k=1}^{\infty} z^{k} \cos (k \theta)
$$


one obtains for $l \geqq 1 \operatorname{Tr} U_{l}(R)=2 \cos (l \theta)$ when $\operatorname{Det} R=1$ and $=0$ when $\operatorname{Det} R=-1$. Furthermore, $\operatorname{Tr} W(R)=\operatorname{Det} R$. Thus the integral (5.4) or (5.12) reduces to

$$
\frac{1}{4 \pi} \int_{0}^{2 \pi}(2 \cos l \theta)^{2} d \theta=1
$$

as was to be shown. Of course, in this rather trivial case Proposition 5 can be verified directly without the aid of the general method, indeed, it reduces to the obvious statement that $L_{12}=l\left(E_{\sigma_{+}}-E_{\sigma_{-}}\right)$.

The case $v=3$ is that of ordinary quantum mechanics, and the result is well known. We present it here for the sake of completeness, partly as a pedagogical exercise, but also to prepare for the new and interesting cases $v \geqq 4$.

The two components of $\mathrm{O}_{3}$ are the subgroup $\mathrm{SO}_{3}$ of those $\mathrm{R \in O}$ with Det $R=1$, and its coset with respect to reflections consisting of those $R \in O_{3}$ with $\operatorname{Det} R=-1$. The canonical form of $R \in O_{3}$ is

$$
R=\left(\begin{array}{ccr}
\cos \phi & \sin \phi & 0 \\
-\sin \phi & \cos \phi & 0 \\
0 & 0 & \pm 1
\end{array}\right) \quad(\operatorname{Det} R= \pm 1)
$$

where $0 \leqq \phi \leqq \pi$, i.e. each $R \in O_{3}$ is equivalent by conjugation in $O_{3}$ to one of the forms (5.17). Functions (as the $p_{l}$ ) that depend only on the conjugacy class can be regarded as depending on $\phi$ and the \pm sign, and their integral with respect to Haar measure is expressed by

$$
\frac{1}{\pi} \underbrace{\pi}_{\text {Det } R=1} \ldots \sin ^{2} \frac{\phi}{2} d \phi+\frac{1}{\pi} \underbrace{\pi}_{\operatorname{Det} R=-1} \ldots \sin ^{2} \frac{\phi}{2} d \phi .
$$

From (5.17) one obtains

$$
\frac{1-z^{2}}{\operatorname{Det}(1-z R)}=\frac{1 \pm z}{1-2 z \cos \phi+z^{2}}, \quad(\operatorname{Det} R= \pm 1)
$$

whence upon expanding in powers of $z$

$$
\operatorname{Tr} U_{l}(R)= \begin{cases}\frac{\sin \left(l+\frac{1}{2}\right) \phi}{\sin \frac{1}{2} \phi} & (\operatorname{Det} R=1) \\ \frac{\cos \left(l+\frac{1}{2}\right) \phi}{\cos \frac{1}{2} \phi} & (\operatorname{Det} R=-1) .\end{cases}
$$

Also from (5.5) $\operatorname{Tr} W(R)=1 \pm 2 \cos \phi(\operatorname{Det} R= \pm 1)$. The integral to be evaluated is then

$$
\begin{aligned}
& \frac{1}{\pi} \int_{0}^{\pi}(1+2 \cos \phi) \frac{\sin ^{2}\left(l+\frac{1}{2}\right)}{\sin ^{2} \frac{1}{2} \phi} \sin \frac{1}{2} \phi d \phi \\
& +\frac{1}{\pi} \int_{0}^{\pi}(1-2 \cos \phi) \frac{\cos ^{2}\left(l+\frac{1}{2}\right) \phi}{\cos ^{2} \frac{1}{2} \phi} \sin ^{2} \frac{1}{2} \phi d \phi \\
& \quad=\frac{1}{2 \pi} \int_{-\pi}^{\pi}(1+2 \cos \phi)(1-\cos (2 l+1) \phi) d \phi \\
& \quad= \begin{cases}1 & (l \geqq 1) \\
0 & (l=0) .\end{cases}
\end{aligned}
$$

This completes the proof of the Lemma for $v=3$. 
Similar calculation could be made for the higher orthogonal groups $O_{v}$ $(v=4,5, \ldots)$. The major complication is in the formula, corresponding to (5.14) and (5.18) above, for the Haar-integral in terms of rotation angles (the curious reader is referred to Murnaghan [4], Chapt. IX, for details). Fortunately, the required integrals have been computed already. Issai Schur, in one of his pioneering papers on the representation theory of orthogonal groups [5], proved the following elegant theorem: If $1 \leqq m \leqq v$, then

$$
\begin{aligned}
\int_{O_{v}} & \prod_{j=1}^{m} \operatorname{Det}\left(1-z_{j} R\right)^{-1} d h(R) \\
= & \prod_{1 \leqq j \leqq k \leqq m}\left(1-z_{j} z_{k}\right)^{-1} .
\end{aligned}
$$

In other words

$$
\int_{O_{v}} p_{l_{1}} p_{l_{2}} \ldots p_{l_{m}} d h(R)
$$

is the coefficient of $z_{1}^{l_{1}} z_{2}^{l_{2}} \ldots z_{m}^{l_{m}}$ in the multi-power series expansion of the rational function on the right hand side of (5.22). The integral (5.12) to be calculated is a sum of six terms which are all of the form (5.23) with $m=3$ and $m=4$. Their calculation can be simplified by grouping terms appropriately and omitting factors from the product $\prod_{1 \leqq j \leqq k \leqq 4}\left(1-z_{j} z_{k}\right)^{-1}$ which make no contribution. Thus it is found that $\int p_{1}^{2}\left(p_{l}-p_{l-2}\right)^{2} d h(R)$ is the coefficient of $\left(z_{1} z_{2}\right)^{l}$ in the expansion of $\left(1+2 z_{1} z_{2}\right)\left(1-z_{1} z_{2}\right)^{-1}$, that is

$$
\int_{O_{v}} p_{1}^{2}\left(p_{l}-p_{l-2}\right)^{2} d h(R)=\left\{\begin{array}{ll}
1 & (l=0) \\
3 & (l \geqq 1)
\end{array} \quad(v \geqq 4) .\right.
$$

Similarly, $\int p_{2}\left(p_{l}-p_{l-1}\right)^{2} d h(R)$ is the coefficient of $\left(z_{1} z_{2}\right)^{l}$ in the expansion of $\left(1+z_{1} z_{2}\right)\left(1-z_{1} z_{2}\right)^{-1}$, that is

$$
\int_{O_{v}} p_{2}\left(p_{l}-p_{l-2}\right)^{2} d h(R)=\left\{\begin{array}{ll}
1 & (l=0) \\
2 & (l \geqq 1)
\end{array} \quad(v \geqq 3) .\right.
$$

Subtracting, one finds (5.12). This completes the proof of the lemma and with it, Proposition 5.

\section{Concluding Remarks}

We are now in a position to construct models of "interacting spins" analogous to those considered by Lieb [1]. Let $\mathbf{v}=\left(v_{1}, v_{2}, \ldots v_{n}\right)$ and $\mathbf{l}=\left(l_{1}, l_{2}, \ldots . l_{n}\right)$ be finite sequences, and let $\mathfrak{S}_{v, 1}=\mathfrak{G}_{v_{1}, l_{1}} \otimes \mathfrak{G}_{v_{2}, l_{2}} \otimes \ldots \otimes \mathfrak{G}_{v_{n}, l_{n}}$. For $1 \leqq r \leqq n$ and $1 \leqq j \leqq k \leqq v_{r}$ let $L_{j k}^{r}$ denote the copy of $L_{j k}$ that operates on the $r^{\text {th }}$ factor of $\mathfrak{G}_{v, \mathrm{l}}$. Let $P=P(\xi)$ be a real polynomial in the variables $\xi=\left(\xi_{12}^{1}, \xi_{13}^{1}, \ldots, \xi_{12}^{n}, \xi_{13}^{n}, \ldots\right)$ with the property that no term in it contains more than one factor $\xi_{j k}^{r}$ with a given $r$ (the "normal case" of Lieb [1]). The quantum mechanical model is defined by the Hamiltonian $H_{\text {quant }}^{v, l, P}=P(L)$, that is, by replacing $\xi_{j k}^{r}$ in $P$ by $L_{j k}^{r}$. The associated partition function is $Z_{\text {quant }}^{v, l, P}=d_{v, 1}^{-1} \operatorname{Tr} \exp \left(-H_{\text {quant }}^{v, 1, P}\right)$, where $d_{v, 1}=\operatorname{dim} \mathfrak{G}_{v, 1}$. The classical analogue of this 
model has the configuration space $\mathbf{M}=M_{v_{1}} \times M_{v_{2}} \times \ldots \times M_{v_{n}}$ whose points are $n$ tuples $\boldsymbol{\sigma}=\left(\sigma_{1}, \sigma_{2}, \ldots \sigma_{n}\right)$ with $\sigma_{r} \in M_{v_{r}}$. The energy function $H_{\text {class }}^{v_{1}, \mathbf{1}, P}(\boldsymbol{\sigma})$ is obtained by replacing $\xi_{j k}^{r}$ in $P$ by $l_{r} m_{j k}\left(\sigma_{r}\right)$, symbolically $H_{\text {class }}^{v, I, P}(\boldsymbol{\sigma})=P(\operatorname{lm}(\boldsymbol{\sigma}))$. The associated partition function is $Z_{\text {class }}^{\mathbf{v}, \mathbf{l}, P}=\int_{\mathbf{M}} \exp \left(-H_{\text {class }}^{\boldsymbol{v}, \mathbf{l}, P}(\boldsymbol{\sigma})\right) d \boldsymbol{\mu}(\boldsymbol{\sigma})$ where $\boldsymbol{\mu}$ is the product measure. The cases considered by Lieb correspond to $v=(3,3, \ldots, 3)$.

Proposition 6. $Z_{\text {class }}^{v, 1, P} \leqq Z_{\text {quant }}^{v, 1, P}$.

Proposition 7. $Z_{\text {quant }}^{v, 1, P} \leqq Z_{\text {class }}^{v, 1+v-2, P}$.

Here $\mathbf{l}+\boldsymbol{v}-2=\left(l_{1}+v_{1}-2, l_{2}+v_{2}-2, \ldots, l_{n}+v_{n}-2\right)$. The proofs are exactly as in Lieb's paper, with notational changes only.

It will not escape the reader that when $v=(2,2, \ldots, 2)$ one has $Z_{\text {quant }}=Z_{\text {class. }}$. This is no miracle, indeed it is a quite trivial and obvious fact. The point is that the distinction between "quantum" and "classical" disappears in this case and becomes a matter of mere notation : both models are just a fancy way of describing the well known Ising model. From the quantum point of view, there is only one spin-component $L_{12}$ for each degree of freedom, having the two eigenvalues $\pm l$, there is no non-commutativity in the model. From the classical point of view, the configuration space $M_{2}$ is just the union of two isolated "points" (the two orientations of $\mathbb{R}^{2}$ ) and the spin-variable is $\operatorname{lm}_{12}(\sigma)= \pm l$ for the two points $\sigma=\sigma_{ \pm} \in M_{2}$. Our embedding of the Ising model as the extreme case of two sequences, one quantum, one classical, makes only official what every physicist knows, that the Ising model can be thought of in either of these two ways.

The inequalities of Propositions 6 and 7 can be used, exactly as in Lieb's work, to prove limit theorems as the quantum number $l \rightarrow \infty$ (but $v$ remains constant). Because of the nature of the inequalities the limit $l \rightarrow \infty$ must be done through an arithmetic sequence of difference $v-2$, then does one obtains the monotonic, interlacing sequences of quantum (resp. classical) partition functions which are used in the proof of the limit theorems.

\section{References}

1. Lieb, E.: Commun. Math. Phys. 31, 327 (1973)

2. Hochstadt, H.: The functions of mathematical physics. New York: Wiley 1971

3. Vilenkin, N.Ja.: Special functions and the theory of group representations. Providence: Am. Math. Soc. 1968

4. Murnaghan, F.D.: The theory of group representations. New York: Dover 1963

5. Schur, I.: Sitz. Preuss. Akad. Wiss. 1924, p. 297. Also Gesammelte Abhandlungen, Vol. II, p. 460. Berlin, Heidelberg, New York: Springer 1973

Communicated by E. Lieb

Received January 11, 1979 